Article

\title{
"Parknerships" for Sustainable Relevance: Perspectives from the San Francisco Bay Area
}

\author{
Elizabeth E. Perry $^{1, *}$, Lydia A. Kiewra ${ }^{1}$ (D), Meghan E. Brooks ${ }^{2}$, Xiao Xiao ${ }^{1}$ \\ and Robert E. Manning ${ }^{1}$ \\ 1 Rubenstein School of Environment and Natural Resources, University of Vermont, Burlington, VT 05405, \\ USA; lydiakiewra@gmail.com (L.A.K.); xiaoxiaonju@gmail.com (X.X.); rmanning@uvm.edu (R.E.M.) \\ 2 College of Agriculture and Life Sciences, University of Vermont, Burlington, VT 05405, USA; \\ mbrooks3@uvm.edu \\ * Correspondence: elizabeth.perry@uvm.edu
}

Received: 12 March 2018; Accepted: 9 May 2018; Published: 15 May 2018

\begin{abstract}
Parknerships" (park partnerships) are an innovative means of enhancing people's connections with conserved spaces and stories, drawing on the combined strengths of multiple organizations. As a specific type of collaboration, a parknership is focused among parks-related organizations sharing common goals of building positive experiences for individuals, the community, and the environment. The need for parknerships is heightened in complex settings like urban areas, and with national organizations concerned with local relevance, such as the National Park Service (NPS). Although parknerships have emerged as a crucial mode of local connection and are increasingly highlighted in park guidance, scant information exists on what mechanisms contribute to their long-term, multi-effort success. We seek to address this by investigating what elements contribute to a sustainable and successful parknership. To frame this inquiry, we drew from the concept of relevance and framework of collective impact. Using semi-structured interviews $(n=14)$ with NPS and partners in the San Francisco Bay Area (an urban area with rich cultural diversity and long environmental history), we elicited understanding of sustainable parknerships. Participants emphasized the importance of the parknerships' context, process, and goal. Consideration of these intersectional themes may be critical to sustainable, relevance-related collaborations among parknerships.
\end{abstract}

Keywords: relevance; urban; collaboration; qualitative; sustainability; collective impact; adaptation; partnership; National Park Service

\section{Introduction}

Protected area management agencies have been at the forefront of the response to the rallying call for increased sustainability practices. Indeed, the balancing act of sustainability [1] is embedded in the mission of the National Park Service (NPS): not only to conserve natural and cultural resources, but also to provide for the enjoyment of these resources "in such manner and by such means as will leave them unimpaired" for future generations also to enjoy [2]. The NPS and related agencies have the potential to implement policy and practice to lessen today's impacts in preference for long-term conservation. In doing so, they serve as leaders in sustainable practices, steering societal changes by making such practices publicly available and accessible [3]. Although notable facets of implementation have included in-park infrastructure and consumption, one with potentially broader resonance is community partnerships. This is where "parknerships" can form. Parknerships are collaborations (formal and informal) specifically among parks-related organizations that share common goals of building positive experiences for individuals, the community, and the environment. Parknership engagements contribute to multiple aspects of urban sustainability. 
In particular, parknerships related to increasing the relevance of a park to its local community (potential stewards) may enhance the park's long-term sustainability. Parknerships often bring together novel and diverse participants and activities, continuously energizing efforts for involved, inclusive, and resilient stewardship connections. Thus, they may strengthen aspects of sustainability within parks. Given that the scope of relevance extends beyond any one organization, the collective impact model provides a framework for goal-focused collaboration [4]. However, relevance-driven collaborations framed by collective impact considerations have rarely been examined, in terms of what may contribute to their long-term success and sustainability.

The need for parknerships is heightened in urban areas, where there is both greater community diversity and imperative for park managers to seek viewpoints and assistance from their many cityscape neighbors [5]. Sustainable efforts, however, do not happen instantaneously; they take time to cultivate and flourish. Working toward a sustainable product demands a sustainable process. One means by which to examine the sustainability of the urban relevance-focused parknership process is through the collective impact $(\mathrm{CI})$ framework.

In this investigation, we sought to elicit aspects of sustainable, relevance-driven urban parknerships, incorporating the CI framework. Although past research has focused on elements of successful collaborations, fewer inquiries have concentrated on successful parknerships and their sustainable processes. To better understand elements of these sustainable, park-centric relationships with local communities and their interdependent functionality, we conducted a program of qualitative inquiry with NPS personnel and community partners in the San Francisco Bay Area, CA, USA (known as the Bay Area in this context). Parknerships in this setting are long-established, generally viewed as successful and sustainable, and utilize the CI framework as a common ground for implementation. This examination provides context for the success of an urban parknership, identifies potentially case-specific and more transferrable attributes of sustainable parknership processes, and highlights integrations of $\mathrm{CI}$ and relevance frameworks.

\section{Background}

We centered this investigation on what contributes to the long-term success and sustainability of parknerships. As such, we have framed our inquiry around considerations of relevance and CI, especially in their application to park management and community conservation collaboratives.

\subsection{Relevance}

A long-noted issue with park visitation, especially in NPS-managed areas, is that although these places are preserved for all, they are not visited by all. In particular, minority racial and ethnic groups have been chronically underrepresented [6-8]. Expanding upon the intergenerational equity emphasis of both sustainability and the NPS mission [9], relevance aims for inclusive practices that broaden and deepen these settings' and stories' resonance, usefulness, or applicability to people's own experiences and quality of life $[10,11]$. In this way, relevance is an unending process of connecting two entities (in this case, organizations and individuals) through a shared goal(s) [12]. The NPS is working on enhancing relevance agency-wide, especially by emphasizing potential connections between urban NPS sites and populations. For example, its Urban Agenda, a centennial initiative, emphasizes the local context by focusing on adjacent communities as potential visitors and promotes a "culture of collaboration" mindset in the NPS to actively engage local partners in this continuous quest for relevant interactions [13].

Collaborative measures in urban areas can have impact on the perceived relevance of parks to people, and thus on the sustainability of these endeavors. The incorporation of diverse community perspectives is crucial $[14,15]$. For example, partners managing the Rivers of Steel National Heritage Area in Pittsburgh have been able to integrate important components of the site (e.g., conservation, interpretation, recreation, economic revitalization) with a localized, community-based focus emphasizing the importance of the heritage area's significance [13]. In this instance and in others, 
having NPS locations in the heart of racially and ethnically diverse urban centers, and involving a partner model in its efforts, allows for increased awareness, visitation, and community benefits citywide [16,17]. Similarly innovative, inclusive, and community-sourced projects may allow the NPS to enhance their relevance to the multitude of cultural groups within the population who use parks in differing ways $[18,19]$. For example, research within the NPS has increasingly examined narratives of non-dominant groups and contested histories [20-22].

Parks have the ability to transform urban spaces into urban places, drawing people in to experience special features and activities that reflect a city's image and personality [23]. To sustain their place on the cityscape, it is vital that these areas are accessible and inviting to locals as well as destination tourists [24]. These areas need to be viewed as places for everyday leisure, which is another perspective on relevance [24-26]. The NPS has recognized that revitalizing urban neighborhoods and industrial infrastructure, more so than preserving areas and structures in museum-like states, may be an appropriate involvement for the agency in urban revival efforts [27]. This model has been applied to varying degrees of success, with the Presidio of Golden Gate National Recreation Area (GGNRA) being a notable example of continuous-use transformation [5]. Integrating urban parks and related programming into everyday life for urban residents contributes to the relevance of these areas to the locals' lives and to the resilience of these people-park connections over time. As the above examples suggest, when relevance has been examined, the focus tends to be on singular programs or events aimed at forging pathways toward relevance. Less has been forwarded on the important consideration of how recurrent and adaptive interactions and collaborations may sustain pathways toward relevance.

\subsection{Parknerships for Relevance}

Parknerships are a means to enhance and sustain relevance, as working with a variety of partners may enhance NPS operations [28]. NPS parks and programs in urban settings are increasingly involved in parknerships with local communities. Often, these relationships leverage resources to create successful connections between parks and people via nontraditional approaches [29]. Examples are found in as wide-ranging contexts as NPS-city collaborations in San Antonio, Texas, to extend city bike trails and bike sharing programs to San Antonio Missions National Historical Park [30]; joint land ownership in Santa Monica Mountains National Recreation Area and GGNRA [5,31]; and programs such as Park Prescriptions that emphasize parks as places to engage in healthy behavior (e.g., physical activity, meditation, time away from screens) [17,32-34]. In responses to the vision set forth for the NPS' next 100 years in "A Call to Action" [35], the NPS has further emphasized the importance of addressing challenges and engaging urban communities through parknerships as a means to strengthen sustainability and stewardship.

Innovative approaches to parknerships suggests an enhanced sustainability of urban park relevance. Prominent examples include the Los Angeles Urban Rangers' efforts to engage urbanites with all area parks by emphasizing the benefits of all parks rather than one park jurisdiction [36] and the innovative emphasis on the connection between urban parks and personal health to show the relevance to people's daily lives and wellbeing [37]. In urban areas where additional large protected areas are not feasible, innovation has taken shape in the evolution of linear urban greenways to creatively use space and promote connectivity within the social-ecological cityscape [38] and the inclusion of pop-up parks that temporarily open otherwise claimed spaces like city streets to leisure [39].

Parknerships can also be a challenge for the NPS. Navigating flexible and site-specific approaches to relationships is a challenge for the NPS, as context-specific actions in one park are often discarded if they are perceived to be inappropriate for application in all parks $[5,27,40]$. This rigidity is especially obvious in urban settings, where the integrity of the site, resources, or community interactions differ from those in other settings [5]. Beyond institutional support, past research has shown factors such as urban proximity, motivation of individual personnel, and lack of reward to influence public resource management agencies' capacity to collaborate [41-43]. Encouraging sustainable use of NPS urban parks requires site-specific approaches to parknership and community engagement that are at times at odds 
with management policies in other areas (e.g., leashed dogs on urban trails) $[5,40,44]$. In planning for the NPS' future, proactively building institutional capacity for more nimble application of parknerships is recognized, with recommendations that the NPS pursue expansive, adaptive, and dynamic systems planning [27] as well as encourage choice, flexibility, and creativity [35]. One primary means of implementing this systems planning is through nurturing a culture of collaboration among NPS and partner organizations $[13,35]$. Therefore, detailing components of sustainable, relevance-driven parknerships would highlight areas for managers to consider and may encourage a more parsimonious pathway through the bureaucratic rigidity.

\subsection{Collective Impact}

Increasing NPS capacity to promote relevant interactions (especially in urban systems) may not successfully or sustainably occur if solely on an individual level. These large scale social changes in complex settings require assistance from multiple partners to be implemented. This type of collaboration between various organizations is an example of CI, where there is commitment, coordination, and consensus between members of a diverse group to solve a specific social problem [4]. This framework finds rooting in emergent and adaptive management considerations [45,46]. Collective work can be successfully implemented when considered from a networked systems stance, rather than one that focuses on discrete engagements [47]. The CI model embodies Aristotle's synergistic "The whole is greater than the sum of its parts." through collaborative rather than solo efforts. To promote a successful and sustainable collaboration, research suggests five factors that aid in success: a common agenda, shared measurement systems, mutually reinforcing activities, continuous communication, and backbone organization or infrastructure [4]. When these elements are in place, the groundwork is set for strategically initiating action, organizing for impact, and sustaining action and impact [48-50]. Successful collaboratives also build time delays into the structures and expectations, slowing down when necessary to ensure details of the key components and phases are appropriately addressed and communicated [49].

Collective impact is, of course, part of a larger body of work on assessing collaborations (e.g., [51,52]). However, CI models in particular, and their relationship to building social capacity, have been studied in a variety of resource management contexts. The framework has aided organizational and community responses to invasive plant species in urban parks [53], large scale watershed management [54], boreal forest conservation [55], and preservation of natural and cultural heritage areas [46]. However, when considering CI for relevance, investigations appear limited to non-park contexts and particularly focused on healthcare and marketing (e.g., [48,56,57]). Therefore, contributing to knowledge on how CI has been implemented specifically for park relevance goals would expand understanding about applications of CI and be of tangible value to park managers and their colleagues.

\section{Research Questions}

Considering the potentially interwoven nature of aspects of relevance and CI, especially in the context of urban parknerships, we explored two related questions:

(1) What elements of parknerships contribute to enhancing sustainable park-people connections?

(2) How are these elements functioning as part of a sustainable process?

\section{Methods}

We sought insight to these questions with conservation community leaders in the San Francisco Bay Area. Qualitative case study analysis was chosen due to the exploratory nature of these questions and the opportunity for deep engagement. 


\subsection{Study Area}

Due to the particularity and complexity of parknerships, we sought a case study location and population that could be adequately probed about the sustainability of its parknership processes, particularly around CI [58]. We concluded that the San Francisco Bay Area was a suitable location for this inquiry for two main reasons.

First, the Bay Area is an urban area rich in cultural diversity (over 7 million inhabitants) and therefore efforts at park relevance must be multifaceted and responsive. Multiple studies have examined how the conservation community interacts with the local community to forge and maintain relevant park-people connections $[59,60]$. These examinations have primarily focused on describing current and desired relevance linkages. The collaborative methods by which the conservation community works toward these linkages have been detailed to a lesser extent, though multiple organizations and the NPS promote CI as a primary framework for parknership work.

Second, the Bay Area also has a long history of environmental engagements. It has a large percentage of conserved lands (24\%) [61] and houses many organizations acknowledged as innovative leaders in conservation and public engagement [62]. Since GGNRA's establishment in 1972, the NPS has long recognized the importance of parks for the people [5]. Local residents have mostly responded favorably, showing high support and use levels of the NPS and other agency parks (East Bay Regional Parks, The Nature Conservancy, etc.) [62]. This history of land use and public involvement in an innovative urban setting has led to notable instances of experimenting (and often succeeding) with means to connect urban residents to these places [5,62].

We focused on a set of NPS-centric parknerships that collaboratively strive for enhanced relevance with the local community. This bounded case interactions and allowed opportunity for deep examination about a specific realm of sustainable parknerships rather than a broad overview [58]. In the Bay Area, the NPS and partners routinely use CI as guidance for parknership development and maintenance. As the emphasis of this investigation is on eliciting mechanisms by which partners work collaboratively to reach for relevance and sustainability goals, the NPS and its partners in the Bay Area were chosen as the case.

\subsection{Recruitment}

We compiled a purposeful sample of potential participants through discussions with a local NPS informant who has worked extensively with parknerships and collaborative communities $[63,64]$. Additional, non-represented participants were recruited during the data collection process through an iterative process of examining areas where there were fewer perspectives [64]. All potential participants were recruited via email; 15 of the 17 contacts agreed to participate ( $88 \%$ response rate). Both contacts who declined participation provided alternate contacts who had already been identified and recruited. The 15 participants were all currently managers, partnership specialists, and/or public outreach officers. Five (33\%) were NPS park superintendents and division chiefs from parks around the Bay Area, most of whom have long work histories with the NPS there. The other $10(67 \%)$ were in similar positions of leadership and influence at six organizations (all with a physical presence in the Bay Area) representing a range of NPS partnerships conditions (e.g., formal agreements, informal collaborations). Three organizations had formal NPS agreements, including work done on collaborative site-ownership, art installations, and community engagement programs and volunteers. The other three had informal NPS collaborations that included promoting natural areas across the Bay Area, creating outdoor recreation programming for racial/ethnic minority affinity groups, and seeking public input on plans for diverse recreation opportunities in NPS and other parks. We included multiple perspectives from two organizations that have a substantial and formalized partnership agreement with GGNRA for two main reasons: (1) the unique agreement structure in place between these organizations and the NPS is not common throughout the NPS; and (2) these organizations are sufficiently large to have subdivisions and thus perspectives were captured from a variety of departments. 


\subsection{Interviews}

We conducted 14 face-to-face interviews in June 2014. One interview was with two individuals from the same organization, as they requested this format to build on each other's contributions. The interviews ranged 35-95 $\mathrm{min}$, averaging $65 \mathrm{~min} /$ participant. We audio-recorded the interviews (with consent) and maintained detailed field notes and researcher memos, consistent with qualitative inquiry [64]. Throughout the interviews and especially after the tenth interview, we heard the same names, examples, and language used to describe Bay Area parknerships, indicating we had approached saturation for this case [64]. The semi-structured interview centered on topics of urban park character, local relevance, parknership collaboration, and nurturing and enhancing collaborative potential. Three questions asked were pertinent to this particular investigation:

1. The National Park Service has promoted "relevance" and a "culture of collaboration" as two priority areas for growth, especially in urban areas. What does each of these mean to you/your organization?

2. Collaborations can occur formally or informally, and with goals that can be explicitly stated or implicitly understood. When thinking about this potential diversity of collaborations, what collaborations for relevance exist between Golden Gate National Recreation Area and other/your organization(s)?

3. Golden Gate National Recreation Area is often upheld as an example of numerous and long-standing collaborations in a national park setting. On the day-to-day level, what successes and challenges have you/your organization encountered in the ongoing process of these types of collaborations?

\subsection{Data Analysis}

Our primary analytic objective was to determine what processes and outcomes collaborators identified as influencing sustainable progress toward the goal of park-people relevance. These articulations of place and process are often embedded within stories of personal experience [65]. Thus, we examined interview transcriptions, field notes, and researcher memos in this light. From these materials, we first generated a field-sourced code list (i.e., inductive analysis) [64]. We refined the codes to a final list of concept categories pertinent to sustainability, relevance, collaborations, NPS interactions, and implementation strategies. We then undertook a second round of coding, structured by this list and aided by qualitative data analysis software [66]. Altogether, 819 passages were coded under these criteria. Afterward, we looked across interviews to identify coding patterns and groupings of larger thematic categories related to sustainability and adaptive change [66]. Throughout this process, we engaged in continual processes of maintaining inter-coder reliability and sharing in analysis and interpretation [66]. Quotes presented in this manuscript have been lightly edited for enhanced readability with care taken to retain intended meaning.

\section{Results}

Our analysis found that sustainable parknerships in the Bay Area are guided by consideration of three main areas: the context, the process, and the goal. Understanding the place-based characteristics of the region has allowed for collaborations to center efforts that are specific and sensitive to the social-ecological system. Within this context, parknerships have molded intersectional processes that balance stability, adaptation, and change. In particular, three facets of this process have been emphasized: (1) establishing a supported structure, (2) cultivating a collaborative mindset, and (3) embracing the unknown. All three, as well as their intersections, are infused with creativity, variety, and flexibility. The sustainability of these processes within this context relies on a clear definition of the overarching goal of relevance. In this manner, the context, the process, and the goal are intertwined in elements of sustainable parknerships. This is depicted in Figure 1 and described more fully below. 


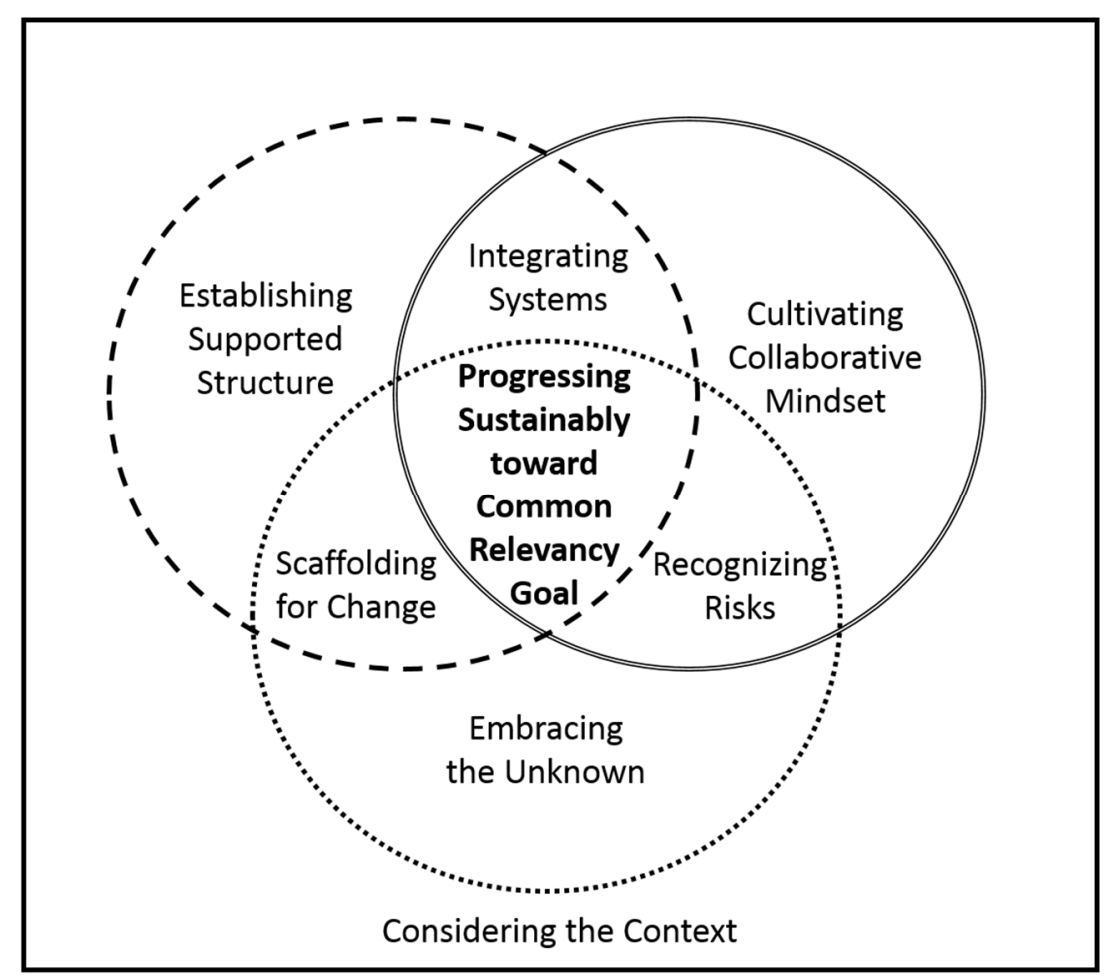

Figure 1. Conceptual model of the intersectional elements of context, processes, and goal contributing to sustainable, relevant parknerships.

\subsection{The Context}

All participants emphasized the unique nature of the Bay Area, implying a place-based understanding and framing of specific parknership aspects. NPS and community partner organizations alike mentioned aspects of the social-ecological system that shaped their consideration of relationships and directions to pursue. In some instances, park lands were juxtaposed with the built environment, as areas of natural respite and escape. Cultural parks, such as Rosie the Riveter WWII Home Front National Historical Park, and opportunities for cultural interpretation elsewhere (e.g., visitor centers, ranger-led programs), were also cast as specific places and instances for reflection and contemplation. These expressed benefits of respite, escape, reflection, and contemplation echo those found in outdoor recreation more broadly and suggest that parks are an integral component to achieving these personal benefits in the Bay Area context. Although the identity of these park places on the natural/cultural spectrum was clearly delineated, it was by no means phrased as separate from the area's other social and ecological features. Instead, the majority of participants spoke of how sites managed by the NPS and others are embedded in the city and enmeshed in a continuous conversation with other interests. This implies not only a recognition of the integrated natural and cultural elements of the urban area but also that these elements are interrelated.

Beyond the physical attributes, participants described the Bay Area setting in terms of its social history, populations, cultures, and concerns. They characterized the occupied landscape as diverse and constantly changing. As one participant said, "Diversity breeds diversity", indicating that heterogeneous areas tend to attract even more heterogeneity. In the interviews, we heard an incorporation of a temporal scale and the increasing complexity of relationships that history brings to current and proposed interactions. Participants repeatedly labeled the Bay Area as an area of social complexity, where the present and future desires of the entire population could be better understood by people who explicitly focus time on learning about the roots of people and policy in the area. Considering the cultures and concerns of the area, parknerships would be able to nurture 
context-appropriate means of striving for relevance goals. This idea of appropriate means within the Bay Area's unique, coupled social-ecological conditions were captured by one participant who described the context as follows:

Well, I think this is an eco-tone, right? 7.5 million people on the edge of this open space and coastal area. The coolness of the Pacific Ocean and the heat of the central coast coming together, creating fog. Serpentine rock. There's a whole unusual thing, elements of an eco-tone. It's being around in a vibrant Bay Area-culturally, intellectually, environmentally. So all those things are part of the dynamic ... Part of being an urban setting is you need to grow and it's a matter of how you do that.

Perhaps in part because of the diverse resources and dynamic of the Bay Area, the area is able to attract innovative and engaged leaders at the helm of parknership organizations. Five respondents evocatively called these leaders sparkplugs. Respondents stressed that these energetic individuals, attracted to this context by the opportunity to further park-people relationships, emphasize the importance of the social sciences in park management, such as commissioning social science studies related to park relevance and then creatively looking at means to implement findings. Examples include re-configuring picnic table arrangements to accommodate large family gatherings, appealing to the stated park leisure patterns of Hispanic/Latino visitors.

\subsection{The Process}

Elements of parknership collaboration processes for enhanced relevance centered on structure, communication, and growth. These elements also embody a time continuum, with building foundations, sharing regularly, and taking chances for improvement as relationships matured. These interrelated components require robust functioning in all sectors for a sustainable, goal-focused process. Given that the process centers on actions, we have correspondingly named the components as such: establishing supported structure, cultivating collaborative mindset, embracing the unknown, and considering the intersections of these three.

Establishing Supported Structure. A common process theme was the need for establishing a supported structure on the organizational and collaborative levels. Participants mentioned that this structure on an organizational level was imperative, with the need for internal training and prioritization of intra-organizational issues (as an NPS employee stated, "tend to the home fires") before considering any external collaborative undertakings. In this way, parknerships were phrased somewhat rigidly, as something that could not be leapt into, but rather needed a formalized curriculum and queue number in the "To Do" list. Building from this internal structure, collaboratives were mentioned as also somewhat rigidly structured and but supported in a variety of ways. This framing of collective efforts encompassed both formalized partnerships and elements of Collective Impact language, as well as informal partnerships (i.e., as-needed and on-demand collaborations) and allusions to collaborative frameworks structuring and nurturing interactions.

Certain elements of structure were repeatedly mentioned as vital components. For example, having defined roles was a crucial consideration in participants' involvement in relevance-related collaboratives. Flexibility was encouraged throughout these defined roles, but having a known capacity set and an ability to act on it within interactions was a fundamental point of structure. NPS and partner personnel defined roles by acknowledging an organization's strengths and limitations. As one participant noted:

We're not doing the work but we're this kind of central backbone, providing support and coordination.

We're not doing a lot of the heavy lifting-we're not taking 100,000 people out every year onto our parks. We're not protected land ourselves but we're coordinating in the middle.

Being a NPS-centric study, it is not surprising that all participants mentioned the NPS' central role in collaborations for relevance. Most of the time (all of the partners and half of the NPS staff), this centrality was coupled with an acknowledgement of the power differentials that come with a 
federal land management player and a diverse collection of national, regional, local, civic, and ad-hoc organizations. Power differential was at times expressed negatively, with the continual challenge of avoiding or breaking down institutional rigidity in the NPS. More often, we heard this difference as either a neutral, inherent part of the assumed structure of the collaborations or a positive opportunity for combining strengths on multiple levels. With statements such as "The NPS doesn't do that. It takes community organizations to come in and do that promoting." resource distribution differences are understood and leveraged as advantages. Participants recognized that this area of rigidity within the structure necessitates flexibility in other areas. They work toward this need for flexible areas with multiple, diverse parknerships.

These power differentials and the range of parknership types within the network also suggested that variety within the structure presents further opportunity for creativity. With the diversity of players involved in relevance-focused collaborations, there are multiple spheres of overlapping partners and the opportunity for sustainable rejuvenation through known means for new players to enter and leave the system. For example, routine and publicized coordination sessions allow for updates on who is moving on from a joint endeavor and who has been identified as a potential collaborator through networking. In considering both the stability and flexibility of structure, these collaboratives can transcend the personality-based attributes of dynamic individuals who can spark parknerships but potentially not pass on such enthusiasm to their successors. Instead, the partners may be able to institutionalize multifaceted approaches to promoting sustainable parknerships.

Cultivating Collaborative Mindset. Cultivating a collaborative mindset focuses on co-growth through routine communication and involvement. Experiences and viewpoints related to communicating, seeking assistance, aligning activities, and sharing in process and product alike were the most common throughout the interviews. Passages coded as such represented more than $25 \%$ of the total coded passages. They also averaged the most instances per interview. Elements of a collaborative mindset included routine, considerate communication; building each other up; seeking each other's assistance; and sharing in process, products, and measurements.

These elements take on a personal nature as well, stepping away from the collaboration at hand to support organizations and individuals in their other functions. These actions show a genuine appreciation for others' work and function as a means to better understand local interactions. Some participants have found that these relationships have an outsized positive impact on enhancing current and future collaborations, as entities are more known as being part of the community. We found general agreement that although the NPS may not have done this to the degree that other organizations have in the past, it is an area of recent growth for the NPS. One NPS participant, discussing how the agency can foster collaborative goodwill throughout the community, noted:

They need just as much support as we need. If we can support them, when they need support, then they're gonna support us when we need it. So, show up, be part of the community. Be present, even when you think you don't need to or you don't have the time or whatever it might be ... And it can't only be when it's to your benefit. Because if it is, people will know it. They're gonna know it.

Mutually reinforcing activities occur throughout the collaborative process. It takes a maintained sense of curiosity to seek these activities and flexibility to be able to continuously share in the process. Multiple participants mentioned that a means to do so was to "share unripe ideas" with partners and constituents, soliciting feedback throughout the process instead of just on the finalized product. This can be difficult to implement; sharing ideas before they are ripe puts an organization in a self-administered vulnerable state. Additionally, recognizing and mutually overhauling "stale projects" was mentioned and also requires a certain vulnerability. The reoccurrence of these unique bookend phrases-unripe and stale-indicates that participants had spent substantial time together discussing these issues, building a localized language about project phases, and reinforcing trust among the group to do so. In the spirit of true collaboration and mutually reinforcing activities, opening up to others requires a strong sense of trust. Indeed, all the elements of cultivating a collaborative mindset were mentioned in tandem with ideas of trusting environments and a receptivity to sharing best efforts. 
Personal and institutional trust takes time to build, and parknership collaborations have been in place in the Bay Area for decades. Communicating openly about the expectations of trust has allowed newer organizations and newer parknerships (e.g., Healthy Parks Healthy People collaboratives with the health industry) to participate in this culture of trust and sharing, seeking assistance from novel places and at all stages. The trust in a respectful culture of sharing has therefore allowed for parknerships that are open to different approaches, configurations, and viewpoints, instead of just different final products.

One challenge alluded to with a collaborative mindset was how to retain connections to multiple, similar collaborators. For example, when one organization routinely communicates and shares deeply with another, there is a lessened need to keep even perfunctory communications with a redundant organization. Although this was posed as a challenge, it was also mentioned as a reason why keeping a diversity of partners within the known system was a benefit; choosing which ties to deepen, and which to keep at more basic levels of interaction, creates a greater opportunity for all organizations and connections to remain in the process despite any duplicative functions.

Embracing the Unknown. The final main process elicited in our interviews centered on an ability to embrace the unknown. These leaps of faith may energize collaborations with new efforts, approaches, and unexpected synergies. Naturally, the process is challenged by the potential for failure in new endeavors and of over-extending/over-reaching. We repeatedly heard of piloting projects as a specific means by which taking chances on new opportunities for relevance-related parknerships can be encouraged despite this challenge. Three participants expressed this embrace by describing parknerships as a petri dish where cultures that thrive in the mix are allowed to develop and become part of the process, such as with allowing a community garden at GGNRA that now holds coveted and deeply cared for plots.

Even visionary leaders hesitate to commence large-scale petri dish efforts if there is a chance for these efforts to not move toward the goal of relevance. Hence, buffer room for trial (and potential failure) has been built into these parknerships through piloting. Piloting initiatives allows for collaboratives to test new projects on a smaller scale before enlarging the initiative to its full scale. The diversity of abilities and guidelines with other members of the collaboratives means that many times, pilot projects may be collaboratively imagined but implemented mainly by these non-NPS entities. One organization saw this as their primary focus. In absorbing this idea of piloting on behalf of the entire collaborative, they saw themselves (and other organizations corroborated this) as an innovation think tank that can use the parknership setting to pilot park practices and see if they can be scaled-up and attract/retain an audience. For example, the idea of sustainable food options at park-based concessionaires was a significant change to the current system. To see if this idea was accepted and could potentially be expanded, one park within GGNRA, Muir Woods National Monument, piloted this type of concessions. Lessons learned from this effort were consistently presented among invested organizations and then shared widely in a planning document that other parks could implement.

\subsection{Intersectionality}

Beyond being discrete elements, we heard examples of intersections among the three main processes for sustaining relevance-focused parknerships. These intersections are also characterized by creativity and variety, which allow for adaptive means of collaborating.

Integrating Systems. Establishing supported structure and cultivating collaborative mindset overlap in instances of integrating systems. Integrating systems refers to a collaborative structure that is functioning, mainly through the synchronicity of its parts. In this case, we heard that this integration is made possible by communication, trust, and sharing. For example, messaging to communities is done in a cohesive manner, lessening the chance for contradictory means of encouraging community outreach and participation in planning processes. Relevance efforts routinely consider social and ecological system impacts, such as PresidiGO, a natural gas-fueled public transportation option to parks for less affluent communities, and providing sustainable food options at park-based concessionaires. 
The ability for the collaborative to respond on a systems-level to community input is also a form of integration. When a proposal set forth as a vision for GGNRA's Crissy Field did not receive a favorable response, the coordinated and diverse roles of the organizations and their deep communication allowed them to pull from a variety of strengths within the network, or as an NPS formal partner stated: "step up, spend a lot of time and resources, and put forward an alternate vision of what this area could look like, something that the public could get behind."

Recognizing Risks. Cultivating collaborative mindset and embracing the unknown overlap when recognizing risks. Taking leaps is necessary for growth but can be disastrous if participants are not aware of the undertakings. This results in a heightened level of transparency and communication required in these instances. It does not automatically translate to full support or involvement, but instead relates to the acknowledgement of novel means to explore the depth of relevance within the community. Transparency may also assist in finding others within the collaborative willing to support that leap as well. One participant highlighted the communication of risks as:

You're willing to take a risk and if you can find a partner that's willing to take a risk, you can gauge the level of success of that risk. You always have to know the risk that's involved when you take a risk.

Scaffolding for Change. Finally, embracing the unknown and establishing supported structure overlap with scaffolding for change. This process area is one that balances form and flexibility, allowing for growth and change by implementing overarching guidelines rather than codifying specific interactions and exacting best practices. It is when organizations rely on the basic foundations and known pathways throughout the collaborative system but are not bound solely to a prescribed set of interactions to enhance relevance. A prominent example is found in the visionary leadership of the NPS and organizations around the Bay Area. NPS staff in the Bay Area tend to stay long-term in their positions, allowing for an enhanced institutional memory and a wealth to draw upon when considering new and modified approaches. Incorporating long-standing leadership with newer players enhances the potential for diverse and creative new efforts while staying rooted in knowledge gained from past attempts. Participants mentioned that leadership qualities are encouraged in staff of all levels, so that all interactions have professional elements and the opportunity to build leadership capacity throughout the system.

\subsection{The Goal}

Applying these intersecting processes with consideration to the context is a means for parknerships to move sustainably toward their relevance goal. As relevance is an ideal that is strived for yet never fully reached, sustainable efforts are key. An important consideration is that the goal must be adequately defined and consistently monitored. Because relevance is an elusive goal, bringing it into a contextualized focus is imperative; it necessitates a community-based rather than organization-based focus. As one participant said, it requires a "very intentional knitting of a park and a community together." With this definition of relevance as external to any one organization, parknerships must continuously broaden their efforts beyond their individual agencies. In the Bay Area, this has been done by promoting all parks and park-like interactions (e.g., cultural institutions), rather than just the properties of a sole entity. We found participants eager to promote opportunities and experiences for engagement even if these were not on lands they managed or were in formal agreement with. For example, a shared calendar of park fitness/wellness events across many jurisdictions has been embraced as a promotion of the Healthy Parks Healthy People concept for parks and people alike. This willingness to broaden the collaborative intent to the entire conservation community exemplifies a functioning and contextualized parknership process with a common goal. Although organizations still retain their individual identities in these parknerships, a cohesive identity is encouraged through the articulation of park relevance as a landscape-spanning concept rather than a particular landscape.

In summary, we found that the context, intersectional process, and goal are fundamental considerations in sustainable parknerships in the Bay Area. Figure 1 captures these relational findings. 
In this model, participant-sourced processes relate to a balance of structure, communication, and adaptation, intersecting with each other and infused with creativity and diversity. This process must be considered within the overarching context. Combined, the process and context adequately define the common goal and provide guiding principles for resilient, relevance-focused urban parknerships.

\section{Discussion}

Collective impact provides basic components of successful collaborations but does not explicitly encourage practitioners to consider elemental conditions of creativity and variety. Yet, the goal of relevance necessitates creative and varied approaches. These considerations assist in fostering adaptable framing that retains core structural support, expects routine communications, and legitimizes leaps of faith. In considering the intersections between frameworks for collaboration and sustainability (i.e., CI and relevance), these components, and their managerial and theoretical implications, are highlighted.

Although specific CI terminology was used a limited number of times, participants alluded to aspects of the framework embedded in their interactions. We found that the five aspects of CI were part of a larger structure of context, process, and goals. Furthermore, the five aspects were expressed mainly in two areas of the process: establishing supported structure and cultivating collaborative mindset. This presence supports previous work on how collective identity through conversation is a precursor to effective inter-organization collaboration [67]. Additionally, we believe that the absence of components in embracing the unknown hints at room for further opportunity for CI model refinement and implementation.

In structured roles and interactions, the ability to take calculated leaps adds a creative dynamic that may be key to not only defining parknerships but also sustaining their ability to grow and adapt [68]. These attributes have similarities to two baseline conditions for successful CI implementation (influential champion and urgency for change) [49] but go beyond them in scope. Whereas these two attributes have been noted as important starting conditions, they do not explicitly contribute to sustaining the collaboration once underway. The incorporation of piloting projects, looking creatively for diverse and deep connections, having flexible pathways for partners to enter/leave the system, and providing room to fail within the collaboration all are examples of additional considerations to the CI model that may enhance the sustainability of the endeavor. This may be especially true in cases where the goal, like relevance, is ever over the horizon and long-term adaptable efforts are essential.

Collective impact requires a contextualized approach for success [4], and participants did frame their collaboration discussions within this overarching consideration of place. Place includes all elements of the system, and participants emphasized this with their mentions of the unique social-ecological system of the Bay Area. The Bay Area is consistently held as an example of where parknerships have worked [5], in part to its uniqueness. Although, indeed, the Bay Area is unique in many ways, we argue that the participants' referral to the region's special nature demonstrates more of a careful consideration of the context than of an unrepeatability in other areas. Personnel staying in their positions for a lengthy time, visionary leadership, leadership building throughout positions, and a welcoming of diverse perspectives are all ways in which this parknership has been able to intimately know its surroundings. This may be a guiding principle for successful sustained parknerships in all the unique areas beyond the Bay Area: afford members the opportunity to truly know and engage with the social-ecological context.

There are many ways in which collaborative roles may be defined in collective efforts. What is most important is that these roles are defined [69]. Although individual organizations certainly house a variety of skill sets, the role of the participant is usually strongly defined in a collaboration $[4,69]$. It is interesting, therefore, how participants expressed collaborative roles that, although defined, also contained numerous references to flexibility and adaptability. Along with the emphasis on entrepreneurial spirit among diverse players, we believe that this is a strong merging point of $\mathrm{CI}$ and relevance. The stress on creativity and diversity was a novel contribution to the CI discussion. In this 
regard, it parallels components of the adaptive change cycle. These flexible and diverse parknership collaborations fit with visions of a more resilient and sustainable society [9]. In this envisioning, sustainable societies not only encompass structures of various scales and degrees of centralization to persist and adapt accordingly but are also valued as necessities for people's response, cooperation, and coordination [9]. The CI framework considers how to work toward a goal effectively; incorporating the relevance-associated attributes of flexibility, diversity, and creativity that participants mentioned is a means of considering how to simultaneously work toward a goal sustainably.

Further integrating CI, relevance goals, and sustainable pathways is the acknowledgement of complex, adaptive systems. Instead of trying to halt any changes in the institutional processes of collaborations or in the audiences with whom they are trying to connect, the parknerships described instead recognize change as a constant. This recognition is coupled with actions to counter disruptions, attempting to reposition the collaborative and its members to respond to the changes while retaining a core identity [9]. With the emphasis on co-growth, fostered by both structure and chance, the parknership investigated exhibits emergent learning in a complex context. This adaptability in learning and interaction among the organizations and their social-ecological environment has been previously noted in contexts such as CI work with community participation in tourism planning [70]. It suggests an ability to embed reflection and subsequent redirection into the parknership process. This not only enhances the overall resilience of the effort but also provides more opportunity for success in meeting the goal.

\section{Conclusions}

In this study, we explored the interwoven nature of relevance and CI in the context of an urban parknership. In particular, we focused our inquiry on the elements of parknerships that contribute to sustainable park-people connections and how these elements function as part of a sustainable process. As a result of our qualitative interview analysis, we found that a clear consideration of the context, process, and goal of parknerships is crucial to sustainable, relevance-related collaborations. The interrelated dynamic among these components, especially the process components (i.e., establishing supported structure, cultivating collaborative mindset, embracing the unknown, and intersections thereof), was highlighted as a main means by which relevance-related efforts are sustained. In examining where CI language was and was not represented throughout this model for sustainability, we found areas for managerial and theoretical focus. Namely, areas to add creativity and diversity of approach must be prioritized if the continuous journey toward relevance will be undertaken collaboratively through parknerships.

\section{Limitations}

This work has both limitation and opportunity for transferability. Our work captures the balance of both nimble and expansive parknership structures and their relationships to relevance-driven CI. There are, however, limitations to this study. The diversity of conserved land management in the Bay Area necessitates that parknerships function well and holistically. The sharing of power and identity seen here may not be so harmonious in areas where there are fewer entities and/or more privately-owned entities. We acknowledge that other methods may further elicit a more cohesive view of parknerships, such as focus groups and questionnaires from a greater breadth of partners. Furthermore, there are other models and frameworks for collaboration beyond CI, such as multi-criteria decision analysis [71] or participatory action research [72]. Framing the case with any of these models, or intersections among them, may have provided a different framework for analysis. However, as CI has been an integral component of multiple organizations' planning efforts within this case, using this particular framing was context-appropriate and managerially-useful. The concept of relevance is also broad. By keeping the intent on local relevance, we constrained the investigation to a certain type of connection. Although the case study approach requires careful consideration of context, our conceptual model of findings in the Bay Area may be a guiding principle for points to consider in collaborative undertakings in 
general and those where sustainable process is necessary in particular. As collaborative governance and parknership models are increasingly turned to for resource management and steward engagement, models that incorporate effective and sustainable pathways will be of use for site-specific adaptation.

\section{Further Research}

This investigation has opened interesting lines of future research. The applicability of the conceptual model on sustainable parknerships to other areas, or even more discrete projects within the Bay Area, would be a noteworthy refinement. Revisiting these data as part of a longitudinal study may also highlight how parknerships continue to evolve and lend further nuance to the discussion on sustainable pathways toward the ever-shifting goal of relevance. Drawing on organizational theory to examine what allows some parknerships to re-energize and refresh through flexibility, creativity, and risk taking would add dimension to these concepts on another scale within the collaborative efforts. Network theory and social network analyses (qualitative and quantitative) could also probe how CI attributes are expressed in collaboratives depending upon the individuals' relationships and positioning. Our model captured three processes as components, and we represented them with equal weight. Given, however, the disparate rates of expression of some components, considerations of the relative importance of each component and reasons underpinning these weights would advance this model further. When the contributions of $\mathrm{CI}$ are overlaid in the urban context, a deeper understanding of the sustainable nature of parknerships for relevance is illuminated.

Author Contributions: E.P. conceived of the project, conducted the data collection, and coordinated all aspects related to drafting this manuscript. L.K. and X.X. provided conceptual comments and contributed to manuscript revisions. M.B. co-led the analysis and manuscript drafting. R.M. provided editorial guidance and logistical support.

Acknowledgments: We express gratitude to the participants in this study for sharing their time and perspectives with us, the NPS Urban Team for their assistance, B Freas for data entry, and Christina Das for data verification. We would also like to thank the Rubenstein School of Environment and Natural Resources at the University of Vermont for providing a research grant to conduct this work. Finally, we would like to thank the three reviewers and journal editor for their insightful feedback, which has strengthened the manuscript.

Conflicts of Interest: The authors declare no conflict of interest.

\section{References}

1. World Commission on Environment and Development. Our Common Future; Oxford University Press: Oxford, UK, 1987.

2. National Park Service. Organic Act of 1916. Available online: https:/ /www.nps.gov/grba/learn/management/ organic-act-of-1916.htm (accessed on 13 May 2018).

3. Roberts, N.S.; Outley, C. Innovation and resourcefulness: Recruit and retain a diverse staff in the 21st century. Parks Recreat. 2002, 37, 39-47.

4. Kania, J.; Kramer, M. Collective Impact. Stanford Social Innovation Review, 2011. Available online: https://ssir.org/articles/entry/collective_impact (accessed on 13 May 2018).

5. Rothman, H.K. The New Urban Park: Golden Gate National Recreation Area and Civic Environmentalism; University Press of Kansas: Lawrence, KS, USA, 2004.

6. Perry, E.E.; Xiao, X.; Manning, R. Barrier or bridge? The role of transportation in national park visitation by racial and ethnic groups. World Leis. J. 2015, 57, 1-12. [CrossRef]

7. Solop, F.; Hagen, K.; Ostergren, D. Ethnic and Racial Diversity of National Park System Visitors and Non-Visitors Technical Report; Northern Arizona University Social Research Laboratory: Flagstaff, AZ, USA, 2003.

8. Taylor, P.; Grandjean, B.; Gramann, J. National Park Service Comprehensive Survey of the American Public 2008-2009: Racial and Ethnic Diversity of National Park System Visitors and Non-Visitors; United States Department of the Interior, National Park Service: Fort Collins, CO, USA, 2011.

9. Harper, C.L. Environment and Society: Human Perspectives on Environmental Issues, 5th ed.; Pearson: Boston, MA, USA, 2012. 
10. Brewer, M. Research design and issues of validity. In Handbook of Research Methods in Social and Personality Psychology; Reis, H., Judd, C., Eds.; Cambridge University Press: Cambridge, UK, 2000; pp. 3-39.

11. Henderson, K.A.; Walker, G.J. Ethnic and racial research methods. In Race, Ethnicity, and Leisure: Perspectives on Research, Theory, and Practice; Stodolska, M., Shinew, K.J., Floyd, M.F., Walker, G.J., Eds.; Human Kinetics: Champaign, IL, USA, 2014; pp. 21-36.

12. Gorayska, B.; Lindsay, R. The roots of relevance. J. Pragmat. 1993, 19, 301-323. [CrossRef]

13. National Park Service (NPS). Stewardship Institute Urban Agenda: Call to Action Initiative; National Park Service: Washington, DC, USA, 2015.

14. Collins, C. Applying Bakhtin in urban studies: The failure of community participation in the Ferguslie Park Partnership. Urban Stud. 1999, 36, 73-90. [CrossRef]

15. Low, S.M.; Taplin, D.; Scheld, S.; Fisher, T. Recapturing erased histories: Ethnicity, design, and cultural representation-A case study of Independence National Historical Park. J. Archit. Plan. Res. 2002, 19, 282-299.

16. Laven, D.N.; Krymkowski, D.H.; Ventriss, C.L.; Manning, R.E.; Mitchell, N.J. From partnerships to networks: New approaches for measuring US National Heritage Area effectiveness. Eval. Rev. 2010, 34, 271-298. [CrossRef] [PubMed]

17. Tardona, D.R.; Bozeman, B.A.; Pierson, K.L. A program encouraging healthy behavior, nature exploration, and recreation through history in an urban national park unit. J. Park Recreat. Adm. 2014, 32, 73-82.

18. Loukaitousideris, A. Urban form and social context: Cultural differentiation in the uses of urban parks. J. Plan. Educ. Res. 1995, 14, 89-102. [CrossRef]

19. Watson, B.; Ratna, A. Bollywood in the park: Thinking intersectionally about public leisure space. Leisure/Loisir 2011, 35, 71-86. [CrossRef]

20. Coslett, D.E.; Chalana, M. National Parks for New Audiences Diversifying Interpretation for Enhanced Contemporary Relevance. Public Hist. 2016, 38, 101-128. [CrossRef]

21. Schiavo, L.B. "White People Like Hiking" Some Implications of NPS Narratives of Relevance and Diversity. Public Hist. 2016, 38, 206-235. [CrossRef]

22. Shea, M.; Zujewski, M.; Parker, J. Resuscitating the promise. Public Hist. 2016, 38, 129-148. [CrossRef]

23. Heintzman, P. Gatineau Park: Public participation and changing park purposes in the wildland-urban interface. Leisure/Loisir 2010, 34, 375-402. [CrossRef]

24. Johnson, A.J.; Glover, T.D.; Stewart, W.P. Attracting locals downtown: Everyday leisure as a place-making initiative. J. Park Recreat. Adm. 2014, 32, 28-42.

25. Francis, M. Urban Parks as Community Places; Jin, J., Ed.; Chuncheon G5 Project: Chuncheon, Korea, 2006; pp. 87-96.

26. Johnson, A.J.; Glover, T.D. Understanding urban public space in a leisure context. Leis. Sci. 2013, 35, $190-197$. [CrossRef]

27. Knowles, T.; Long, G. Planning a Future for the National Park System: A Foundation for the 21st Century; 2012 National Parks Advisory Board Report; National Park Service: Washington, DC, USA, 2012.

28. Knapp, C.N.; Chapin, F.S.; Kofinas, G.P.; Fresco, N.; Carothers, C.; Craver, A. The importance of multistakeholder engagement in adaptation planning for conserved areas. Ecol. Soc. 2014, 19, 16. [CrossRef]

29. Economides, A. From Open Spaces to Vital Places; Urban Place Consulting Group, Inc.: Long Beach, CA, USA, 2004; p. 20.

30. Sherwood, K.; Murphy, J. Expanding a Municipal Bikeshare System into an Urban National Park Through Community Partnerships City of San Antonio, Texas, and San Antonio Missions National Historical Park. Transp. Res. Rec. 2014, 2453, 54-61. [CrossRef]

31. National Park Service (NPS) Advisory Board. Urban Committee Meeting Notes; National Park Service (NPS) Advisory Board: Washington, DC, USA, 6 June 2014.

32. Baur, J.W.R.; Tynon, J.F. Small-Scale Urban Nature Parks: Why Should We Care? Leis. Sci. 2010, 32, $195-200$. [CrossRef]

33. Institute at the Golden Gate. Park Prescriptions: Profiles and Resources for Good Health from the Great Outdoors. 2010. Available online: http:/ / www.americantrails.org/resources/health/Park-PrescriptionsHealth-Great-Outdoors.html (accessed on 13 May 2018).

34. Liechty, T.; Mowen, A.J.; Payne, L.L.; Henderson, K.A.; Bocarro, J.N.; Bruton, C.; Godbey, G. Public park and recreation managers' experiences with health partnerships. J. Park Recreat. Adm. 2014, 32, 11-27. 
35. National Park Service (NPS). A Call to Action: Preparing for a Second Century of Stewardship and Engagement; National Park Service: Washington, DC, USA, 2013.

36. Bauch, N.; Scott, E.E. The Los Angeles Urban Rangers: Actualizing geographic thought. Cult. Geogr. 2012, 19, 401-409. [CrossRef]

37. Wong, D.; Higgins, C.L. Park rangers as public health educators: The public health in the parks grants initiative. Am. J. Public Health 2010, 100, 1370-1373. [CrossRef] [PubMed]

38. Searns, R.M. The evolution of greenways as an adaptive urban landscape form. Landsc. Urban Plan. 1995, 33, 65-80. [CrossRef]

39. Wilson, J.; Tierney, P.; Kim, M.-S.; Zieff, S.G. Temporary parks? Sunday streets, serving the need for urban outdoor recreation. J. Park Recreat. Adm. 2012, 30, 38-52.

40. National Park Service (NPS). Advisory Board Appendix F: Urban Sub-Group Report; National Park Service: Washington, DC, USA, 2012.

41. McCreary, A.; Seekamp, E.; Cerveny, L.K.; Carver, A.D. Natural Resource Agencies and Their Motivations to Partner: The Public Lands Partnership Model. Leis. Sci. 2012, 34, 470-489. [CrossRef]

42. Seekamp, E.; Cerveny, L.K.; McCreary, A. Institutional, Individual, and Socio-Cultural Domains of Partnerships: A Typology of USDA Forest Service Recreation Partners. Environ. Manag. 2011, 48, 615-630. [CrossRef] [PubMed]

43. Seekamp, E.; Barrow, L.A.; Cerveny, L.K. The growing phenomenon of partnerships: A survey of personnel perceptions. J. For. 2013, 111, 412-419.

44. Tardona, D.R. Promoting companion animal leash compliance on an urban park trail system. Nat. Areas J. 2012, 32, 215-217. [CrossRef]

45. Kania, J.; Kramer, M. Embracing Emergence: How Collective Impact Addresses Complexity. Stanford Social Innovation Review, 2013. Available online: https://ssir.org/articles/entry/social_progress_through_ collective_impact (accessed on 13 May 2018).

46. Nagurka, P.; Orr, J.; Picat, I.; Smith, J.; Thompson-Deahl, C. Collective Impact for Environmental Conservation. Global Impact. 2013, 1-27.

47. Barnes, M.L.; Sharpe, E.K. Looking beyond traditional volunteer management: A case study of an alternative approach to volunteer engagement in parks and recreation. Voluntas 2009, 20, 169-187. [CrossRef]

48. Flood, J.; Minkler, M.; Hennessey Lavery, S.; Estrada, J.; Falbe, J. The collective impact model and its potential for health promotion: Overview and case study of a Healthy Retail Initiative in San Francisco. Health Educ. Behav. 2015, 42, 654-668. [CrossRef] [PubMed]

49. Hanleybrown, F.; Kania, J.; Kramer, M. Channeling Change: Making Collective Impact Work. Stanford Social Innovation Review, 2012. Available online: https:/ /www.keywestchamber.org/uploads/4/6/5/2/ 46520599/channeling_change_pdf-ssi-article-2012.pdf (accessed on 13 May 2018).

50. Lamm, H. Investigating the roles of community foundations in the establishment and sustainability of local college access networks in Michigan. Found. Rev. 2012, 4, 65-76.

51. Duan-Barnett, N.; Wangelin, J.; Lamm, H. Models of social change: Community foundations and agenda setting. Found. Rev. 2012, 4, 84-97. [CrossRef]

52. Ramaley, J.A. Educating for a changing world: The importance of an equity mindset. Metrop. Univ. 2014, 25, 5-15.

53. Dolan, R.W.; Harris, K.A.; Adler, M. Community involvement to address a long-standing invasive species problem: Aspects of civic ecology in practice. Ecol. Restor. 2015, 33, 316-325. [CrossRef]

54. Enloe, S.K.; Schulte, L.A.; Tyndall, J.C. Toward a collaborative approach to watershed management: Lessons learned from the Boone River watershed, Iowa. J. Soil Water Conserv. 2014, 69, 149A-153A. [CrossRef]

55. Reid, R. The Canadian boreal forest agreement: Unlikely allies pursuing conservation and sustainable development in Canada's boreal regions. Philanthropist 2014, 26, 65-73.

56. Fombrun, C.; Astley, W.G. Beyond corporate strategy. J. Bus. Strateg. 1983, 3, 47-54. [CrossRef]

57. Nahlen, B.L.; Low-Beer, D. Building to collective impact: The Global Fund support for measuring reduction in the burden of malaria. Am. J. Trop. Med. Hyg. 2007, 77, 321-327. [CrossRef] [PubMed]

58. Stake, R.E. The Art of Case Study Research; SAGE Publications, Inc.: Thousand Oaks, CA, USA, 1995.

59. Roberts, N.S.; Chitewere, T. Speaking of justice: Exploring ethnic minority perspectives of the Golden Gate National Recreation Area. Environ. Pract. 2011, 13, 354-369. [CrossRef] 
60. Roberts, N.S.; Suren, A.T. Through the eyes of youth: A qualitative evaluation of outdoor leadership programs. J. Park Recreat. Adm. 2010, 28, 59-80.

61. Rissman, A.R.; Merenlender, A.M. The conservation contributions of conservation easements: Analysis of the San Francisco Bay Area Protected Lands Spatial Database. Ecol. Soc. 2008, 13, 40. [CrossRef]

62. Walker, R. The Country in the City: The Greening of the San Francisco Bay Area; University of Washington Press: Seattle, WA, USA, 2007.

63. Stake, R.E. Qualitative Research: Studying How Things Work; The Guilford Press: New York City, NY, USA, 2010; ISBN 978-1-60623-545-4.

64. Warren, C.A.B.; Karner, T.X. Discovering Qualitative Methods: Field Research, Interviews, and Analysis, 2nd ed.; Oxford University Press: New York City, NY, USA, 2010.

65. Stewart, W. Community-based place meanings for park planning. Leisure/Loisir 2006, 30, 405-416. [CrossRef]

66. Saldana, J. The Coding Manual for Qualitative Researchers, 2nd ed.; SAGE Publications, Inc.: Los Angeles, CA, USA, 2013.

67. Hardy, C.; Lawrence, T.; Grant, D. Discourse and collaboration: The role of conversations and collective identity. Acad. Manag. Rev. 2005, 30, 58-77. [CrossRef]

68. Hardy, C.; Maguire, S. Organizing risk: Discourse, power, and "riskification". Acad. Manag. Rev. 2016, 41, 80-108. [CrossRef]

69. Fallon, F. Strategic collaboration with volunteers in events: Partnering for success. In Collaboration in Tourism Businesses and Destinations: A Handbook; Gursoy, D., Saayman, M., Sotiriadis, M.D., Eds.; Emerald Group Publishing: Bingley, UK, 2015; p. 368.

70. Wray, M. Adopting and implementing a transactive approach to sustainable tourism planning: Translating theory into practice. J. Sustain. Tour. 2011, 19, 605-627. [CrossRef]

71. Dodgson, J.S.; Spackman, M.; Pearman, A.; Phillips, L.D. Multi-Criteria Analysis: A Manual; Department for Communities and Local Government: London, UK, 2009. Available online: http:/ / eprints.lse.ac.uk/12761/ 1/Multi-criteria_Analysis.pdf (accessed on 13 May 2018).

72. Whyte, W.F.E. Participatory Action Research; SAGE Publications, Inc.: Thousand Oaks, CA, USA, 1991.

(C) 2018 by the authors. Licensee MDPI, Basel, Switzerland. This article is an open access article distributed under the terms and conditions of the Creative Commons Attribution (CC BY) license (http:/ / creativecommons.org/licenses/by/4.0/). 